Article

\title{
Subdiffusive Reaction Model of Molecular Species in Liquid Layers: Fractional Reaction-Telegraph Approach
}

\author{
Ashraf M. Tawfik ${ }^{1, *(D)}$ and Mohamed Mokhtar Hefny ${ }^{2}(\mathbb{D}$ \\ 1 Theoretical Physics Research Group, Faculty of Science, Mansoura University, Mansoura 35516, Egypt \\ 2 Engineering Mathematics and Physics Department, Faculty of Engineering and Technology, \\ Future University in Egypt, Cairo 11835, Egypt; mmokhtar@fue.edu.eg \\ * Correspondence: ashroof_tawfik@mans.edu.eg
}

Citation: Tawfik, A.M.; Hefny, M.M. Subdiffusive Reaction Model of Molecular Species in Liquid Layers: Fractional Reaction-Telegraph Approach. Fractal Fract. 2021, 5, 51. https://doi.org/10.3390/ fractalfract5020051

Academic Editor: Trifce Sandev

Received: 7 May 2021

Accepted: 1 June 2021

Published: 3 June 2021

Publisher's Note: MDPI stays neutral with regard to jurisdictional claims in published maps and institutional affiliations.

Copyright: (c) 2021 by the authors. Licensee MDPI, Basel, Switzerland. This article is an open access article distributed under the terms and conditions of the Creative Commons Attribution (CC BY) license (https:/ / creativecommons.org/licenses/by/ $4.0 /)$.

\begin{abstract}
In recent years, different experimental works with molecular simulation techniques have been developed to study the transport of plasma-generated reactive species in liquid layers. Here, we improve the classical transport model that describes the molecular species movement in liquid layers via considering the fractional reaction-telegraph equation. We have considered the fractional equation to describe a non-Brownian motion of molecular species in a liquid layer, which have different diffusivities. The analytical solution of the fractional reaction-telegraph equation, which is defined in terms of the Caputo fractional derivative, is obtained by using the Laplace-Fourier technique. The profiles of species density with the mean square displacement are discussed in each case for different values of the time-fractional order and relaxation time.
\end{abstract}

Keywords: anomalous diffusion; fractional calculus; cold atmospheric plasma; plasma with liquids

\section{Introduction}

Plasma medicine is a multidisciplinary field developed in the last decades by researchers from many fields such as physics, chemistry, biology, biochemistry, engineering, medicine, and many others [1-3]. One of the main targets of plasma medicine is to deliver the use of physical plasmas into hospitals. That can be achieved by development of different plasma sources operating at atmospheric pressure and tissue-tolerable temperatures (known as cold atmospheric plasmas (CAPs)) together with modeling of these plasmas and their surroundings [4,5]. CAPs are used in many applications, such as the generation of reactive species, water treatment, bio-medical applications, and material synthesis [6,7].

One of the unique properties of CAPs is their ability to generate reactive oxygen species (ROS) and reactive nitrogen species (RNS) with high densities at room temperature without heating the gas mixture significantly. The generation of ROS and RNS is well known in some other fields. However, the novelty of CAPs is their ability to produce different collections of these species during the treatment process in a localized manner. Moreover, a fine-tuning for the concentration of the selected species according to the application can occur $[4,8]$. Further, the reactive species have different lifetimes and different reaction rates in the gas phase and in aqueous solutions, and they can be converted to each other.

The interaction between CAPs and biological targets in most cases involves plasmaliquid interaction. Therefore, this interaction is very important in plasma medicine and has been studied by many researchers to understand the behavior of plasma-generated reactive species above and in the liquid, where CAPs can be generated in the top surface of the water, an outer surface of a gas bubble, or even an aerosol droplet in the gas phase. The plasma-generated reactive species can interact with the water surface and generate more reactive species, or they can be diffused directly into the water and hence react with components in the aqueous phase, which makes the plasma-treated water enriched with chemical reactions, and this can be utilized in many biological applications [4,9]. For example, Blackert et al. reported in their work [10], using a dielectric barrier discharge 
(DBD) plasma source in air and above the liquid surface, that ROS in the plasma/gas phase could be transferred to the liquid and react with components in the aqueous phase. $\mathrm{H}_{2} \mathrm{O}_{2}$, as one of the most important plasma-generated reactive species, was reported to be produced by an argon-based radio frequency plasma jet and then diffused to the aqueous phase [11]. Moreover Wende et al. reported that plasma-generated atomic oxygen can initiate chemical reactions in a standard physiological buffer or liquid via reactions with chloride, yielding $\mathrm{Cl}_{2}{ }^{-}$or $\mathrm{ClO}^{-}$. Gorbanev et al. [12] reported that, by using isotopes, $\mathrm{H}_{2} \mathrm{O}_{2}, \mathrm{O}_{2}{ }^{-}, \mathrm{OH}, \mathrm{OOH}$, and $\mathrm{H}$ species in the plasma-treated liquid originate from the gas phase. Lukes et al. also evidenced the generation of some ROS like $\mathrm{ONOOH}, \mathrm{NO}_{2}, \mathrm{NO}$, and $\mathrm{OH}$ radicals and $\mathrm{NO}^{+}$by the air plasma at the gas-liquid interface because of postdischarge processes in plasma-activated liquid [13]. The plasma-generated reactive species can affect many biological targets directly (direct exposure to CAPs) or indirectly through plasma-activated liquid (PAL), where they have a prolonged effect on biological targets ranging from minutes and can reach several days after treatment with plasma [4,14-17].

In previous work, it has been reported that plasma-generated $O_{(g a s)}$ atoms can be diffused to the aqueous solutions and initiate their chemical reactions. Moreover, it was reported that, by using ${ }^{18} \mathrm{O}_{2}$ isotope, the $\mathrm{O}_{(\text {aqueous })}$ atoms react directly with phenol molecules, without dissociating water molecules in intermediate reactions [9]. Additionally, a 2D axially symmetric fluid model of the gas flow and diffusion-convective transport of plasmagenerated reactive species combined with the basic kinetic model of the reaction of $\mathrm{O}$ atoms with $\mathrm{O}_{2}$ molecules has been solved to study the transport and surface reactivity of $\mathrm{O}$ atoms.

From a mathematical point of view, the previous works have introduced the reactiondiffusion equation as a model to describe the diffusive evolution of any molecular species density, which is given by [18-21]

$$
\frac{\partial}{\partial t} u(z, t)=\kappa \frac{\partial^{2}}{\partial z^{2}} u(z, t)+F(u) .
$$

Here, a drifting along the $z$ direction has the probability density $u(z, t)$ and $\kappa$ is the diffusion constant. The last term in the above equation $(F(u))$ represents a reaction function out of each point $z$ (the production or loss probability of species in a liquid layer) such as in $[22,23]$.

The different classes of reaction-diffusion equations are used to model a system combining diffusive motion with reaction. However, physically, such equations imply an infinite propagation speed. In addition, we know that not all species travel inside a liquid layer and reach the target. Therefore, reaction-diffusion equations are not an accurate or optimal description for studying the diffusion of an active molecular species traveling in cluster mediums. To overcome such infinitely fast propagation, a more precise characterization is provided by the reaction-telegraph equations [24,25], which is given by

$$
\tau \frac{\partial^{2}}{\partial t^{2}} u(z, t)+\left[1-\tau F^{\prime}(u)\right] \frac{\partial}{\partial t} u(z, t)=\kappa \frac{\partial^{2}}{\partial z^{2}} u(z, t)+F(u),
$$

where $\tau$ is a characteristic time of the microscopic movement that quantifies the direction of individual molecular species movement (relaxation time), and $F^{\prime}(u)$ denotes the ordinary derivative of a reaction term concerning its argument. With the singular limit $\tau=0$, Equation (2) goes to the reaction-diffusion Equation (1).

Yusupov et al. [26] have used computational techniques (molecular dynamic simulation) to study the behavior of reactive species in a liquid film. In their study, they calculated the mean square displacement (MSD) with the trajectories of different oxygen species $\left(\mathrm{O}, \mathrm{OH}, \mathrm{HO}_{2}\right.$ and $\left.\mathrm{H}_{2} \mathrm{O}_{2}\right)$. They also illustrated different spatial distributions for three kinds of molecular species in the water layer. They found that not all species have the same distribution behavior (classical Gaussian behavior), and the values of the diffusion coefficients are not the same for all species. That means diffusivity is not the same for every molecular species, especially for $O$ radical, which cannot travel deep into the liquid layer (slow-diffusion). Therefore, we will consider a new treatment by suggesting the case of 
subdiffusion, which is more suitable to verify the non-Gaussian distribution and cover all classes of diffusivity (classical and anomalous). Anomalous diffusion is described by nonGaussian statistics, which encompasses probability densities with power-law tails [27-29]. The mean square displacement of a diffusive species is characterized by $\left\langle x^{2}(t)\right\rangle \sim t^{\gamma}$ (non-linear with time). The process is called sub-diffusive when $0<\gamma<1$, while it is called super-diffusive for $1<\gamma<2$.

In recent decades, the fractional differential equations have been developed to describe the anomalous transport process [30-35]. Our attention is directed now to including the sub-diffusive case, which can be obtained by employing a fractional technique. However, using fractional calculus in diffusion problems may differ from one issue to another due to the various definitions of fractional derivatives. In our model, we prefer Caputo's derivative rather than other fractional derivatives because it can provide initial conditions with a physical interpretation for a differential equation of fractional order. We can involve that by replacing the time derivatives in Equation (2) with Caputo's fractional derivative, e.g., refs. [36,37]. The solution of the fractional reaction-telegraph equation gives a useful description of non-diffusive phenomena. It can also be used to illustrate the details of the diffusion process for an evolving particle density function.

In the next section, we obtain an analytical solution to the time-fractional reactiontelegraph equation using the Laplace-Fourier technique. To obtain probability densities compatible with the data obtained by molecular dynamics simulations, the spatial density profiles are predicted for different cases of molecular species transport and this will be illustrated in Section 3. Finally, a summary closes the paper.

\section{Fractional Reaction-Telegraph Model}

In this section, we treat a classical transport theory of plasma-generated ROS to follow their trajectories in a liquid water layer. Introducing a sub-diffusive transport model describes the ROS movement in that layer through generalizing the reaction-telegraph Equation (2) in a semi-finite domain to the time-fractional form e.g., in ref. [38]:

$$
\tau^{\alpha} D_{t}^{\alpha} D_{t}^{\alpha} u(z, t)+[1-\tau(p-r)] D_{t}^{\alpha} u(z, t)=\kappa_{\alpha} \frac{\partial^{2}}{\partial z^{2}} u(z, t)+(p-r) u(z, t) .
$$

The above fractional equation governs the range of density function $u(z, t)$ in a semifinite medium $0<z<\infty$ for $t>0$. Here, we choose $F(u)=(p-r) u$ to represent the case of growth and loss of probability because of the reaction during the transport of molecular species inside a liquid layer. $p u$ is the gain probability after reaction, and $r u$ is the loss probability (absorbed ratio) in reaction with a liquid layer such as given by [9].

In general, the sub-diffusive case of particle motion is described by an equation with a time-fractional derivative (motion of a non-Markovian type) [34,39]. Where a timefractional derivative as in Equation (3) is responsible for introducing a nonlinear time dependence in the mean square displacement of the system, that is instead of a non-linear approach such as is given in [21]. In addition to that, the fractional reaction-telegraph equation can be obtained from the continuous-time random walk [28,39], and it could be formulated in terms of a distributed order derivative [40].

The fractional reaction-telegraph Equation (3) represents a mathematical model for different molecular species movement in a liquid layer as shown in Figure 1. CAPs can be generated between two powered electrodes with a feeding gas composed from a noble gas with a small admixture of molecular oxygen and hence a cocktail of ROS can be generated and even tuned according to the required application [4].

The fractional derivative used here is the Caputo fractional derivative operator [41,42], which is defined in the case of $0<\alpha<1$ as:

$$
D_{x}^{\alpha} f(x)=\frac{1}{\Gamma(1-\alpha)} \int_{0}^{x}(x-\xi)^{-\alpha} \frac{d f(\xi)}{d \xi} d \xi
$$


With Laplace transform [43]:

$$
L\left\{D_{t}^{\alpha} \phi(t), s\right\}=s^{\alpha} \phi(s)-s^{\alpha-1} \phi(0), \quad 0<\alpha \leq 1 .
$$

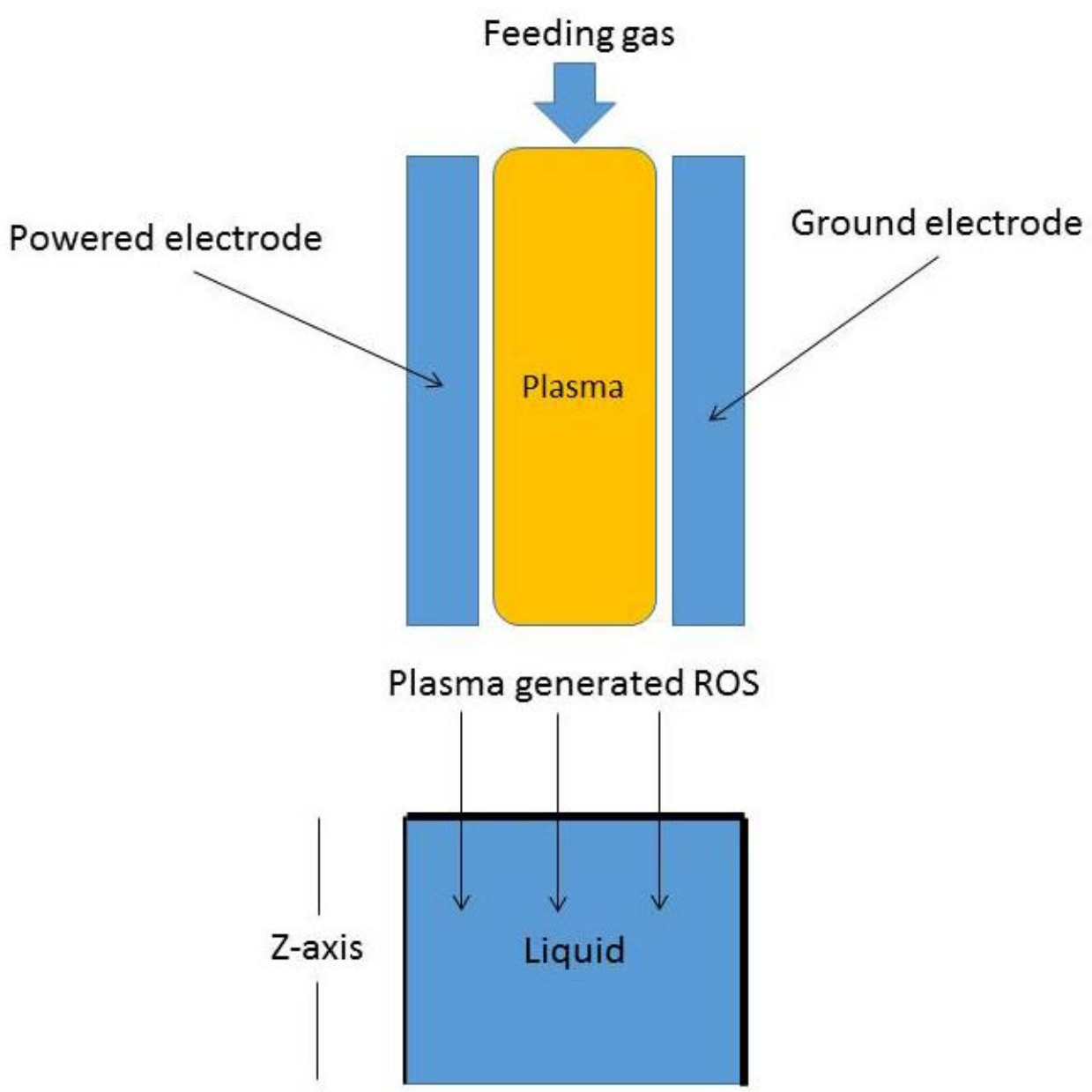

Figure 1. Schematic figure of reactive oxygen species (ROS) interaction with a liquid layer: a representation of molecular species transport in one dimension (semi-finite domain).

Here, the fractional derivative is in the sense of the left Caputo fractional derivative due to the physical causality principle, which means the current value of the probability density function depends on all its previous states. The Caputo fractional derivative with the density gradient is most appropriate to express the fractional Fick law [44]. Moreover, the Caputo fractional derivative is very useful for solving applied problems leading to linear fractional differential equations with accompanying initial conditions in a traditional form instead of the other non-local fractional derivatives (initial conditions written as derivatives of integer order) [45].

The fractional reaction-telegraph equation with the initial condition can be solved using the Laplace-Fourier transforms, with a source point representing the injection of a molecular species in the front of the liquid layer, so that the initial value is given such as in [46]

$$
F(z, 0)=\delta(z), \quad \partial_{t} F(z, 0)=0,
$$

with the assumption that $\left.u^{\prime}(z, t)\right|_{z=0}=0$. 
Taking the Laplace and Fourier cosine transforms to Equation (3), we obtain

$$
u(k, s)=\frac{\omega s^{\alpha-1}}{\tau^{\alpha} s^{2 \alpha}+\omega s^{\alpha}+\kappa_{\alpha} k^{2}+(r-p)}+\frac{\tau^{\alpha} s^{2 \alpha-1}}{\tau^{\alpha} s^{2 \alpha}+\omega s^{\alpha}+\kappa_{\alpha} k^{2}+(r-p)},
$$

where $\omega=1-\tau(p-r)$.

To solve Equation (7), we first rewrite it in an integral form as:

$$
u(k, s)=\int_{0}^{\infty} e^{-\xi(r-p))} e^{-\xi \kappa_{\alpha} k^{2}}\left(\tau^{\alpha} s^{2 \alpha-1}+\omega s^{\alpha-1}\right) e^{-\xi\left(\tau^{\alpha} s^{2 \alpha}+\omega s^{\alpha}\right)} d \xi .
$$

If we consider

$$
\begin{gathered}
g_{1}(k, \xi)=e^{-\xi \kappa_{\alpha} k^{2}}, \\
g_{2}(\xi, s)=\left(\tau^{\alpha} s^{2 \alpha-1}+\omega s^{\alpha-1}\right) e^{-\xi\left(\tau^{\alpha} s^{2 \alpha}+\omega s^{\alpha}\right),}
\end{gathered}
$$

then the solution of Equation (3) is given by

$$
u(z, t)=\int_{0}^{\infty} e^{-\xi(r-p)} g_{1}(z, \xi) g_{2}(\xi, t) d \xi .
$$

To obtain a final form of $u(z, t)$, we must first calculate the function $g_{1}(z, \xi)$, which can be obtained directly by applying the inverse Fourier cosine transform to Equation (9), then

$$
g_{1}(z, \xi)=\frac{1}{\sqrt{\pi \kappa_{\alpha} \xi}} e^{-\frac{z^{2}}{4 \kappa_{\alpha} \xi}}
$$

Second, the function $g_{2}(\xi, t)$ is obtained by applying the inverse Laplace transform to Equation (10):

$$
g_{2}(\xi, t)=L^{-1}\left\{\left(\tau^{\alpha} s^{2 \alpha-1}+\omega s^{\alpha-1}\right) e^{-\xi\left(\tau^{\alpha} s^{2 \alpha}+\omega s^{\alpha}\right)}\right\} .
$$

The above equation can be expressed as

$$
\begin{aligned}
g_{2}(\xi, t) & =L^{-1}\left\{\tau^{\alpha} \sum_{n=0}^{\infty} \frac{\left(-\xi \tau^{\alpha}\right)^{n}}{n !} s^{2 \alpha n+2 \alpha-1} e^{-\xi \omega s^{\alpha}}\right\} \\
& +L^{-1}\left\{\omega \sum_{n=0}^{\infty} \frac{\left(-\xi \tau^{\alpha}\right)^{n}}{n !} s^{2 \alpha n+\alpha-1} e^{-\xi \omega s^{\alpha}}\right\} .
\end{aligned}
$$

Continuing in the same manner as [37], $g_{2}(\xi, t)$ will be given as

$$
g_{2}(\xi, t)=\left(\tau^{\alpha} t^{-2 \alpha} W_{-\alpha, 1-2 \alpha}\left(-\xi \omega t^{-\alpha}\right)+\omega t^{-\alpha} M_{\alpha}\left(\xi \omega t^{-\alpha}\right)\right),
$$

where $W_{v, \mu}$ and $M_{v}$ are the Wright and $M$-Wright functions defined in the Appendix A.

Inserting Equations (12) and (15) into Equation (11), one obtains the solution of the fractional reaction-telegraph Equation (3), which is given by

$$
u(z, t)=\frac{1}{\sqrt{\pi \kappa_{\alpha}}} \int_{0}^{\infty} \frac{1}{\sqrt{\xi}} e^{-\xi(r-p))} e^{\frac{-z^{2}}{4 \kappa \alpha}}\left(\tau^{\alpha} t^{-2 \alpha} W_{-\alpha, 1-2 \alpha}\left(-\xi \omega t^{-\alpha}\right)+\omega t^{-\alpha} M_{\alpha}\left(\xi \omega t^{-\alpha}\right)\right) d \xi .
$$


In the case of $\tau=0$ and $\alpha=1$, the above solution (fractional reaction-telegraph solution) is reduced to the solution of the reaction-diffusion equation in a semi-finite medium, which is given by:

$$
u(z, t)=\frac{1}{\sqrt{\pi \kappa t}} e^{-(r-p) t} e^{-\frac{z^{2}}{4 \kappa t}}
$$

\section{Discussion}

As we mentioned in the introduction, studying the behavior of plasma-generated reactive species in a liquid layer is very important in plasma medicine. Therefore, we have developed a new theoretical model to study the transport of plasma-generated ROS in a liquid layer.

From the solution of the fractional reaction-telegraph Equation (17), we show three parameters that can control the diffusion behavior and the degree of diffusivity. As we can see, the integral given by Equation (17) is evaluated based on the value of the timefractional order $(\alpha)$, relaxation time $(\tau)$ and the reaction rate value $(p-r)$, which means for every value of such parameter, the diffusivity will be changed, which is useful to track and stimulate the different species inside the liquid layer. We address that in detail through Figures 2 and 3.
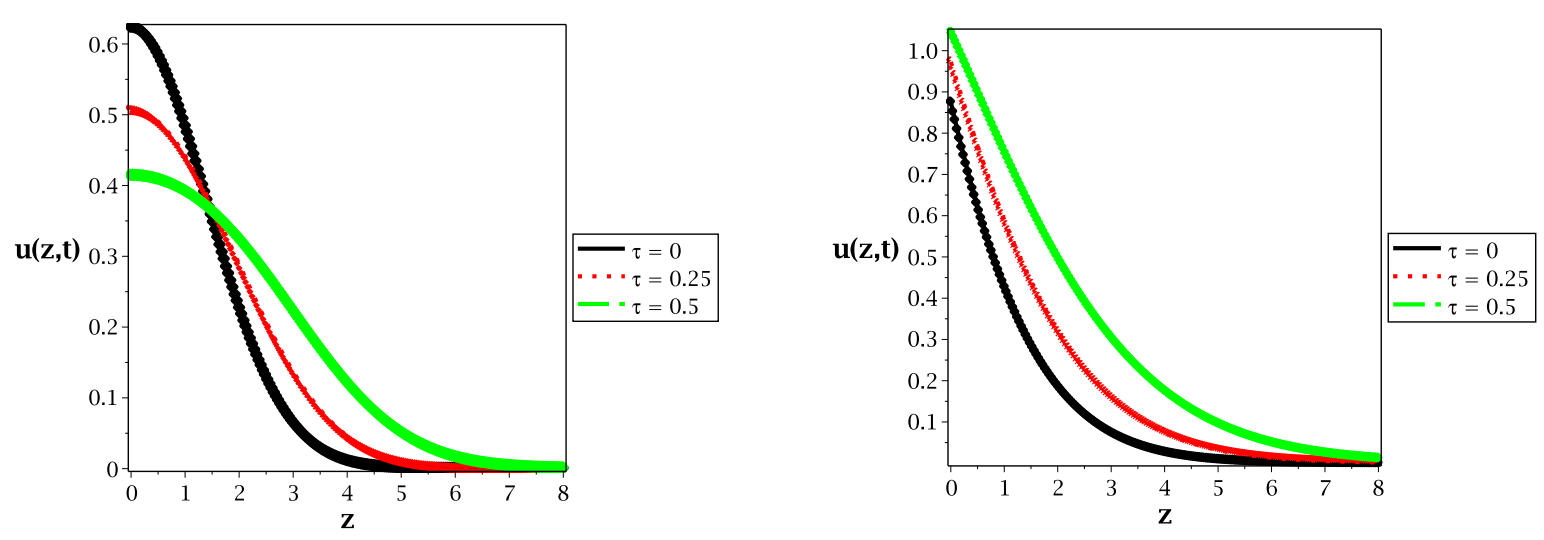

Figure 2. Spatial distribution along the z-axis in the case of $\alpha=1$ (left panel) and $\alpha=1 / 2$ (right panel) for different values of relaxation time $\tau$ at $\kappa=1, p-r=0.1$ and $t=1$.
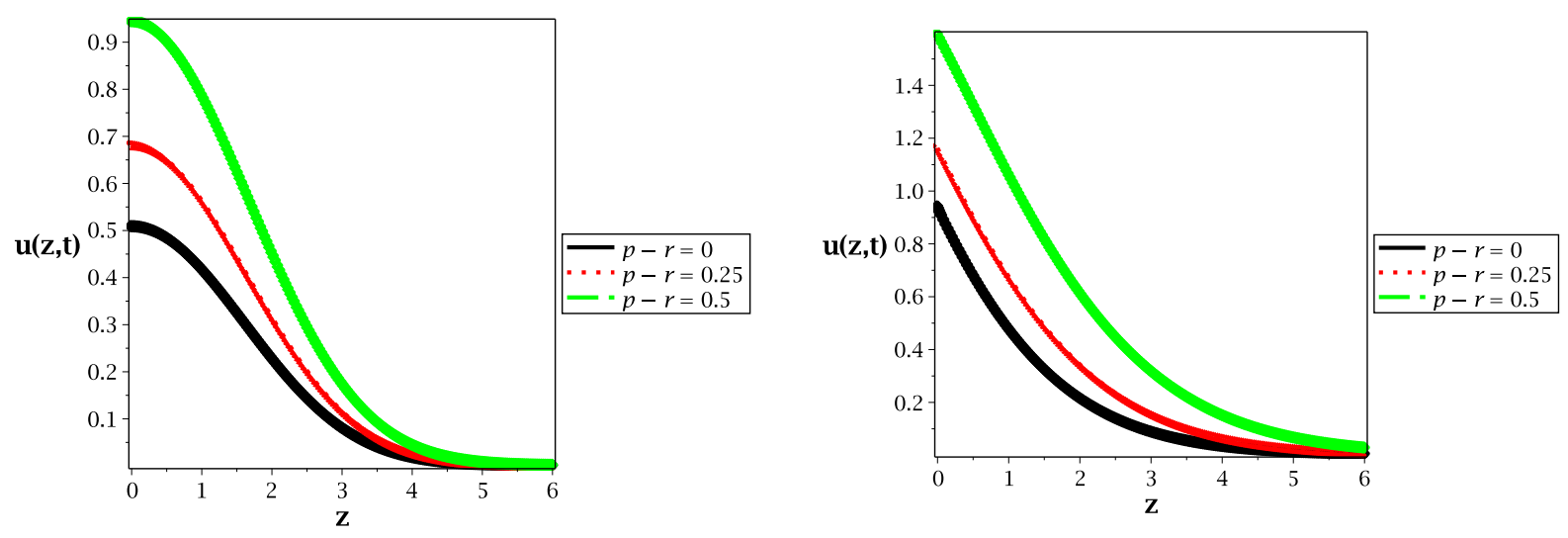

Figure 3. Spatial distribution along the z-axis in the case of $\alpha=1$ (left panel) and $\alpha=1 / 2$ (right panel) for different values of reaction rate $p-r$ at $\kappa=1, \tau=0.2$ and $t=1$.

In Figure 2, we illustrate the spatial distribution of the reactive species for different values of relaxation time along the $z$ axis for the normal diffusive $\alpha=1$ (left panel) and for the sub-diffusive case $\alpha=1 / 2$ (right panel). In Figure 2 we show that changing the value 
of relaxation time implies the speed of propagation in the case of a symmetrical Gaussian distribution at $\alpha=1$ in the left panel and a more shifted non-Gaussian (asymmetrical) distribution with a lower value of time-fractional order (right panel). In addition to that, as a limiting case of study, the solution of the reaction-diffusion equation is illustrated in Figure 2 (black curve at $\tau=0$ ) for the classical case (left panel) and the fractional case (right panel).

Figure 3 presents the spatial distributions of molecular species for different values of reaction rate (gain or loss of probability) in the cases of normal diffusion (left panel) and subdiffusion (right panel). We show that increasing the value of reaction rate extends the peak of probability to a large value, which leads to long-term reactions. Furthermore, at $p-r=0$ (black curves) in Figure 3, the classical and fractional telegraph equations without reaction are illustrated in the left and right panels, respectively.

Moreover, the sub-diffusive behavior (time-fractional case) at $\alpha=1 / 2$ shows through the cusp-shapes of the distribution function, which is suggested for the transport of species such as $\mathrm{H}_{2} \mathrm{O}_{2}$ and $\mathrm{HO}_{2}$. That is due to the contribution of the time-fractional order, which shows fatter tails than the reference case of the Gaussian distribution in the case of $\mathrm{OH}$. This agrees with Yusupov et al. [26], who concluded that the $\mathrm{OH}$ radicals are more rapidly transported than the other two species, and this was validated through calculating the MSD and diffusion coefficients for these species as well.

In addition, the comparison between the diffusion behavior of molecular species is illustrated with a reaction and without a reaction. As we can see, the distributions of all species along the $z$ axis become sharper when we add the reaction term. However, for example, the distribution of species like $\mathrm{OH}$ in ref. [26] decreases the most, and this is in line with its reactivity in an aqueous solution, where it is known to be the most reactive oxygen species [47].

Besides that, one of the significant tools to determine the diffusivity of molecular species traveling inside the water layer is based on illustrating the mean square displacement, such as is given in simulation results $[9,26]$. From our mathematical model here, we can calculate the mean square displacement of a particle density via the inverse-Laplace transform [28]

$$
\left\langle z^{2}(t)\right\rangle=L^{-1}\left[-\frac{\partial^{2}}{\partial k^{2}} u(k, s)\right]_{k=0} .
$$

From the spatial distribution of the three oxygen species illustrated in [26], we show that the reaction does not have a central role in diffusivity. For example, $\mathrm{H}_{2} \mathrm{O}_{2}$ molecules travel without reaction faster than $\mathrm{HO}_{2}$ and slower than $\mathrm{OH}$; however, both form a reaction through traveling in the water layer. Therefore, we can study the typical behavior of mean square displacement in the case of neglecting the reaction terms until the timefractional order and relaxation effects can be demonstrated. In this case, the mean square displacement of the fractional telegraph equation can be expressed by the form:

$$
\left\langle z^{2}(t)\right\rangle=2 \kappa_{\alpha} L^{-1}\left[\frac{s^{-\alpha-1}}{\tau^{\alpha} S^{\alpha}+1}\right],
$$

then,

$$
\left\langle z^{2}(t)\right\rangle=\frac{2 \kappa_{\alpha} t^{2 \alpha}}{\tau^{\alpha}} E_{\alpha, 2 \alpha+1}\left(\tau^{-\alpha} t^{\alpha}\right) .
$$

where $E_{a, b}$ is the Mittag-Leffler function defined in Equation (A4). By following the manner used in [48], the asymptotic behavior can be observed for a large argument

$$
\left\langle z^{2}(t)\right\rangle \sim \frac{2 \kappa_{\alpha} t^{\alpha}}{\Gamma(1+\alpha)},
$$


and for short time limits:

$$
\left\langle z^{2}(t)\right\rangle \sim \frac{2 \kappa_{\alpha} t^{2 \alpha}}{\tau^{\alpha} \Gamma(1+2 \alpha)} .
$$

From Equation (20), we can illustrate the mean square displacement behavior for different molecular species, such as in Figures 4 and 5. In Figure 4, we show the behavior of mean square displacement for different relaxation time values at very short times. From that figure, it can be shown that increasing the value of relaxation time decreases the rate of diffusivity. That means the relaxation time plays an essential role in illustrating the MSD at a short scale. That will be more suitable and effective in fitting our results with the previously published simulations, such as that given by Figure 7 in [26]. Moreover, we compare the reaction-telegraph and reaction-diffusion equations in Figure 5. This confirms the fast speed of propagation in the case of $\tau=0$ (right panel) and the slower propagation with more waiting times of propagation in the case of $\tau=0.5$ (left panel). This will be more realistic for different species such as $\mathrm{H}_{2} \mathrm{O}_{2}$ because it moves slowly inside the water layer and almost reaches the biological targets. Besides that, the $\mathrm{O}$ species already failed to reach the target, and we suggest that this is because of its slow spread (sub-diffusive) with long waiting times inside the liquid layer. Therefore, it is more convenient to describe the motion of different molecular species to introduce the time-fractional order to switch between the normal and slow diffusion. In addition, the relaxation time plays an essential role in regulating the speed of propagation for each molecule and thus covers all forms of transport inside the liquid layer. From this point of view in our brief discussion, we believe now that it is essential to work with the fractional reaction-telegraph instead of the classical reaction-diffusion built up in many numerical simulations.
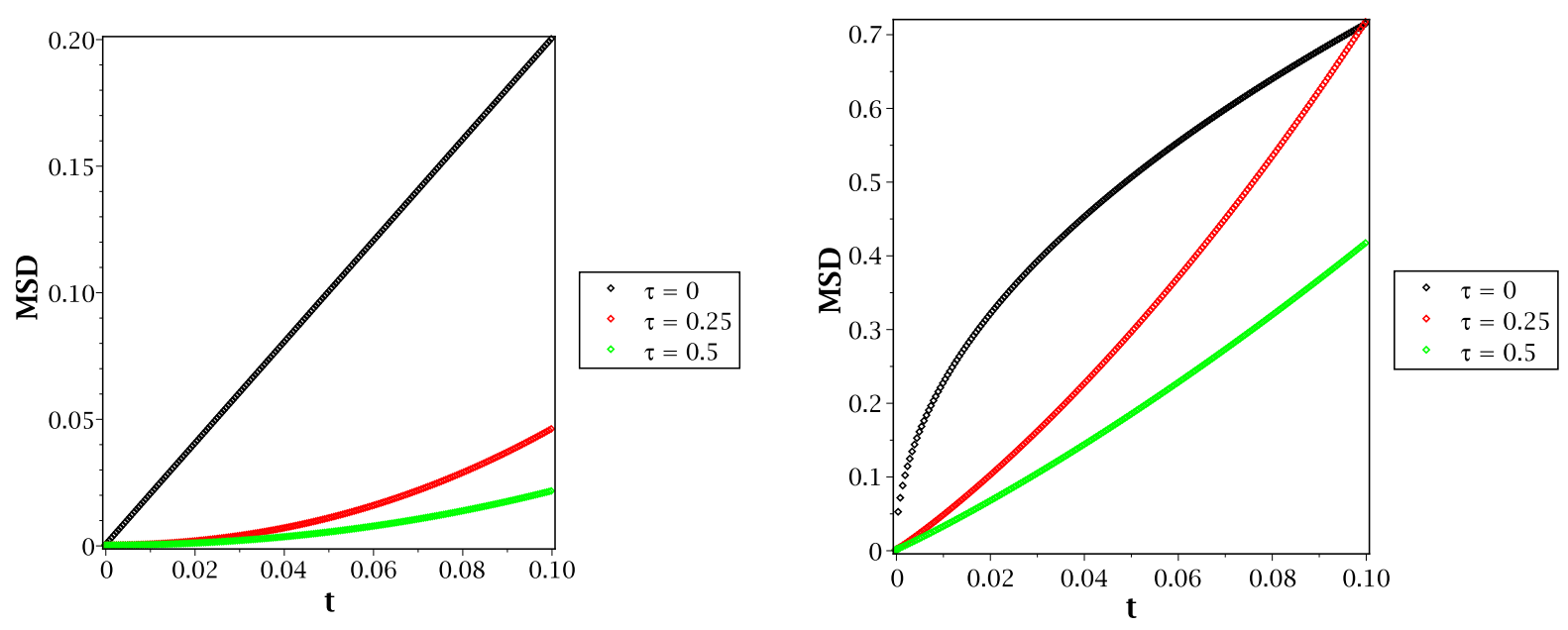

Figure 4. MSD in the case of $\alpha=1$ (left panel) and $\alpha=1 / 2$ (right panel) with different values of relaxation time $\tau$ at $\kappa=1$. 

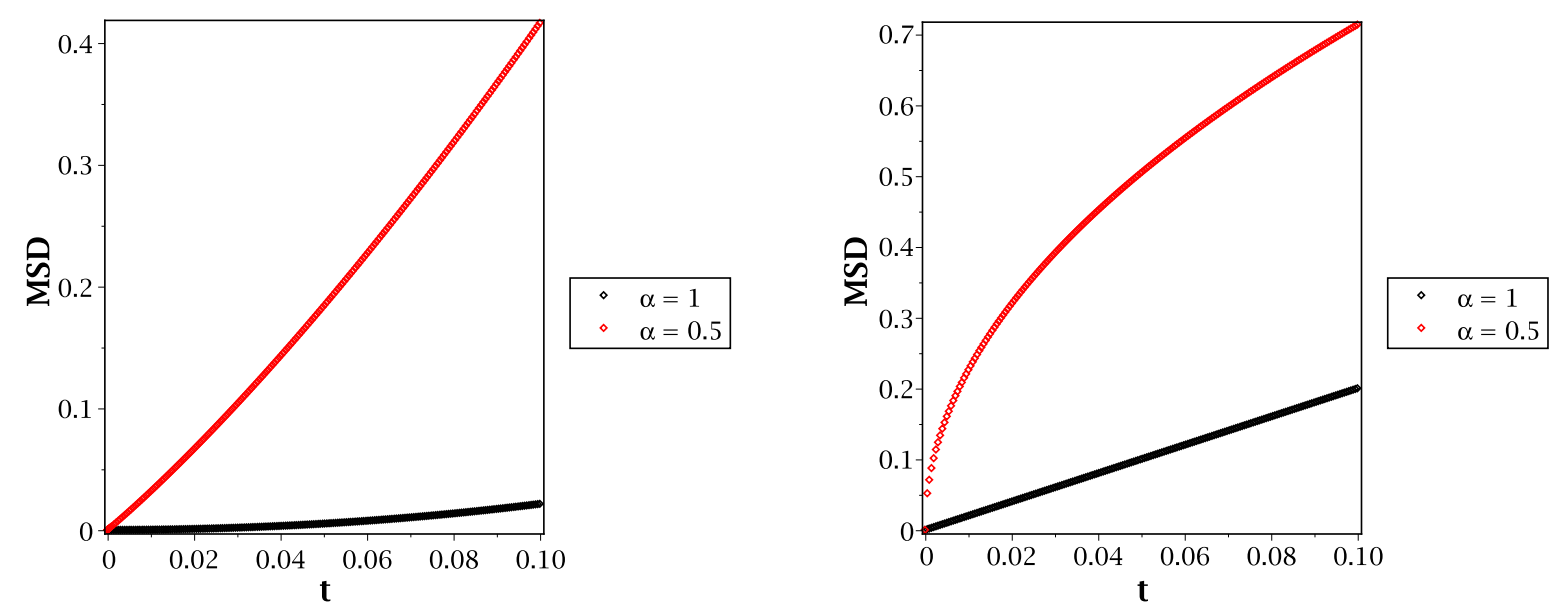

Figure 5. MSD in the case of $\tau=0.5$ (left panel) and $\tau=0$ (right panel) for different values of the time fractional order at $\kappa=1$.

\section{Summary}

This paper has presented some consequences of solving the generalized fractional reaction-telegraph equation using Caputo differential operators. We used the Laplace transform on the time variable and the Fourier transform on the space variable to solve the fractional equation. The solution was given in terms of the computable integral of the Wright function with its auxiliary, and particular cases were recovered. In general, we can deduce that the fractional reaction-telegraph approach yielded more identical predictions for describing the sub-diffusive transport of molecular species, even for a short time, which can give us more information about the transport of the plasma-generated ROS in a liquid layer. Moreover, these findings can help us to understand the reaction of ROS with biological targets, especially those that occurred in a very short time.

Author Contributions: The authors A.M.T. and M.M.H. contributed equally to the conceptualization, methodology, and writing. All authors have read and agreed to the published version of the manuscript.

Funding: This research received no external funding.

Institutional Review Board Statement: Not applicable.

Informed Consent Statement: Not applicable.

Data Availability Statement: Not applicable.

Conflicts of Interest: The authors declare no conflict of interest.

\section{Appendix A}

In this appendix, we present the Wright function with its auxiliary, the so-called $M$-Wright function.

The Wright function denoted by $W_{\lambda, \mu}(x)$ is defined as [49]

$$
W_{v, \mu}(x)=\sum_{n=0}^{\infty} \frac{x^{n}}{n ! \Gamma(n v+\mu)}, \quad v>-1, \mu \in C .
$$

The $M$-Wright function is defined as [50]

$$
M_{v}(x)=W_{-v, 1-v}(-x)=\sum_{n=0}^{\infty} \frac{(-x)^{n}}{n ! \Gamma(-v n+1-v)},
$$


with some explicit representation

$$
M_{1 / 2}(z)=\frac{1}{\sqrt{\pi}} \exp \left(-z^{2} / 4\right) \quad M_{1}(z)=\delta(z-1) .
$$

The Mittag-Leffler function of the second kind [51] is defined as

$$
E_{a, b}(y)=\sum_{m=0}^{\infty} \frac{y^{m}}{\Gamma(a m+b)} .
$$

\section{References}

1. Fridman, A. Plasma Chemistry; Cambridge University Press: Cambridge, UK, 2008.

2. Kong, M.G.; Kroesen, G.; Morfill, G.; Nosenko, T.; Shimizu, T.; Van Dijk, J.; Zimmermann, J. Plasma medicine: An introductory review. New J. Phys. 2009, 11, 115012. [CrossRef]

3. Laroussi, M.; Kong, M.; Morfill, G. Plasma Medicine: Applications of Low-Temperature Gas Plasmas in Medicine and Biology; Cambridge University Press: Cambridge, UK, 2012.

4. Hefny, M.M.; Kairo, A. Experimental Study of Cold Atmospheric Plasma for Plasma Medicine Research and Applications. Ph.D. Thesis, Ruhr-Universität Bochum, Bochum, Germany, 2019.

5. Fridman, G.; Friedman, G.; Gutsol, A.; Shekhter, A.B.; Vasilets, V.N.; Fridman, A. Applied plasma medicine. Plasma Process. Polym. 2008, 5, 503-533. [CrossRef]

6. Bekeschus, S.; Wende, K.; Hefny, M.M.; Rödder, K.; Jablonowski, H.; Schmidt, A.; von Woedtke, T.; Weltmann, K.D.; Benedikt, J. Oxygen atoms are critical in rendering THP-1 leukaemia cells susceptible to cold physical plasma-induced apoptosis. Sci. Rep. 2017, 7, 1-12. [CrossRef] [PubMed]

7. El-Kalliny, A.S.; Abd-Elmaksoud, S.; El-Liethy, M.A.; Abu Hashish, H.M.; Abdel-Wahed, M.S.; Hefny, M.M.; Hamza, I.A. Efficacy of Cold Atmospheric Plasma Treatment on Chemical and Microbial Pollutants in Water. ChemistrySelect 2021, 6, 3409-3416. [CrossRef]

8. Hefny, M.M.; Pattyn, C.; Lukes, P.; Benedikt, J. Atmospheric plasma generates oxygen atoms as oxidizing species in aqueous solutions. J. Phys. D Appl. Phys. 2016, 49, 404002. [CrossRef]

9. Benedikt, J.; Hefny, M.M.; Shaw, A.; Buckley, B.R.; Iza, F.; Schäkermann, S.; Bandow, J. The fate of plasma-generated oxygen atoms in aqueous solutions: Non-equilibrium atmospheric pressure plasmas as an efficient source of atomic O (aq). Phys. Chem. Chem. Phys. 2018, 20, 12037-12042. [CrossRef]

10. Blackert, S.; Haertel, B.; Wende, K.; von Woedtke, T.; Lindequist, U. Influence of non-thermal atmospheric pressure plasma on cellular structures and processes in human keratinocytes (HaCaT). J. Dermatol. Sci. 2013, 70, 173-181. [CrossRef]

11. Wende, K.; Straßenburg, S.; Haertel, B.; Harms, M.; Holtz, S.; Barton, A.; Masur, K.; von Woedtke, T.; Lindequist, U. Atmospheric pressure plasma jet treatment evokes transient oxidative stress in HaCaT keratinocytes and influences cell physiology. Cell Biol. Int. 2014, 38, 412-425. [CrossRef]

12. Gorbanev, Y.; O'Connell, D.; Chechik, V. Non-thermal plasma in contact with water: The origin of species. Chem. A Eur. J. 2016, 22, 3496-3505. [CrossRef] [PubMed]

13. Lukes, P.; Dolezalova, E.; Sisrova, I.; Clupek, M. Aqueous-phase chemistry and bactericidal effects from an air discharge plasma in contact with water: Evidence for the formation of peroxynitrite through a pseudo-second-order post-discharge reaction of $\mathrm{H}_{2} \mathrm{O}_{2}$ and $\mathrm{HNO}_{2}$. Plasma Sources Sci. Technol. 2014, 23, 015019. [CrossRef]

14. Duan, J.; Ma, M.; Yusupov, M.; Cordeiro, R.M.; Lu, X.; Bogaerts, A. The penetration of reactive oxygen and nitrogen species across the stratum corneum. Plasma Process. Polym. 2020, 17, 2000005. [CrossRef]

15. Yusupov, M.; Razzokov, J.; Cordeiro, R.M.; Bogaerts, A. Transport of reactive oxygen and nitrogen species across aquaporin: A molecular level picture. Oxidative Med. Cell. Longev. 2019, 2019. [CrossRef] [PubMed]

16. Van der Paal, J.; Hong, S.H.; Yusupov, M.; Gaur, N.; Oh, J.S.; Short, R.D.; Szili, E.J.; Bogaerts, A. How membrane lipids influence plasma delivery of reactive oxygen species into cells and subsequent DNA damage: An experimental and computational study. Phys. Chem. Chem. Phys. 2019, 21, 19327-19341. [CrossRef]

17. Schneider, S.; Lackmann, J.W.; Ellerweg, D.; Denis, B.; Narberhaus, F.; Bandow, J.E.; Benedikt, J. The role of VUV radiation in the inactivation of bacteria with an atmospheric pressure plasma jet. Plasma Process. Polym. 2012, 9, 561-568. [CrossRef]

18. Hefny, M.M.; Nečas, D.; Zajíčková, L.; Benedikt, J. The transport and surface reactivity of O atoms during the atmospheric plasma etching of hydrogenated amorphous carbon films. Plasma Sources Sci. Technol. 2019, 28, 035010. [CrossRef]

19. Sene, N. Fractional model for a class of diffusion-reaction equation represented by the fractional-order derivative. Fractal Fract. 2020, 4, 15. [CrossRef]

20. Hadeler, K.P. Reaction transport systems in biological modelling. In Mathematics Inspired by Biology; Springer: New York, NY, USA, 1999; pp. 95-150.

21. Boon, J.P.; Lutsko, J.F.; Lutsko, C. Microscopic approach to nonlinear reaction-diffusion: The case of morphogen gradient formation. Phys. Rev. E 2012, 85, 021126. [CrossRef] 
22. Evans, M.R.; Majumdar, S.N. Diffusion with stochastic resetting. Phys. Rev. Lett. 2011, 106, 160601. [CrossRef]

23. dos Santos, M.A. Fractional Prabhakar derivative in diffusion equation with non-static stochastic resetting. Physics 2019, 1, 40-58. [CrossRef]

24. Mendez, V.; Fedotov, S.; Horsthemke, W. Reaction-Transport Systems: Mesoscopic Foundations, Fronts, and Spatial Instabilities; Springer Science \& Business Media: Berlin, Germany, 2010.

25. Alharbi, W.; Petrovskii, S. Critical domain problem for the reaction-telegraph equation model of population dynamics. Mathematics 2018, 6, 59. [CrossRef]

26. Yusupov, M.; Neyts, E.; Simon, P.; Berdiyorov, G.; Snoeckx, R.; Van Duin, A.; Bogaerts, A. Reactive molecular dynamics simulations of oxygen species in a liquid water layer of interest for plasma medicine. J. Phys. D Appl. Phys. 2013, $47,025205$. [CrossRef]

27. Dorea, C.C.; Medino, A.V. Anomalous diffusion index for Lévy motions. J. Stat. Phys. 2006, 123, 685-698. [CrossRef]

28. Metzler, R.; Klafter, J. The random walk's guide to anomalous diffusion: A fractional dynamics approach. Phys. Rep. 2000, 339, 1-77. [CrossRef]

29. Antonio Faustino dos Santos, M. Comb Model with Non-Static Stochastic Resetting and Anomalous Diffusion. Fractal Fract. 2020, 4, 28. [CrossRef]

30. Klages, R.; Radons, G.; Sokolov, I.M. Anomalous Transport: Foundations and Applications; John Wiley \& Sons: Hoboken, NJ, USA, 2008.

31. Datsko, B.; Kutniv, M.; Włoch, A. Mathematical modelling of pattern formation in activator-inhibitor reaction-Diffusion systems with anomalous diffusion. J. Math. Chem. 2020, 58, 612-631. [CrossRef]

32. Compte, A.; Metzler, R. The generalized Cattaneo equation for the description of anomalous transport processes. J. Phys. A Math. Gen. 1997, 30, 7277. [CrossRef]

33. Dos Santos, M.A. Non-Gaussian distributions to random walk in the context of memory kernels. Fractal Fract. 2018, 2, 20. [CrossRef]

34. Sandev, T.; Tomovski, Ž. Fractional Equations and Models; Springer: New York, NY, USA, 2019.

35. Lenzi, E.K.; Evangelista, L.R.; Zola, R.S.; Petreska, I.; Sandev, T. Fractional Schrödinger equation and anomalous relaxation: Nonlocal terms and delta potentials. Mod. Phys. Lett. A 2021, 36, 2140004. [CrossRef]

36. Baleanu, D.; Jassim, H.K. Exact Solution of Two-Dimensional Fractional Partial Differential Equations. Fractal Fract. $2020,4,21$. [CrossRef]

37. Tawfik, A.M. On fractional approximations of the Fokker-Planck equation for energetic particle transport. Eur. Phys. J. Plus 2020, 135, 1-19. [CrossRef]

38. Tawfik, A.M.; Abdou, M.; Gepreel, K.A. An analytical solution of the time-fractional telegraph equation describing neutron transport in a nuclear reactor. Indian J. Phys. 2021, 1-6. [CrossRef]

39. Klafter, J.; Sokolov, I.M. First Steps in Random Walks: From Tools to Applications; Oxford University Press: Oxford, UK, 2011.

40. Chechkin, A.; Gorenflo, R.; Sokolov, I. Retarding subdiffusion and accelerating superdiffusion governed by distributed-order fractional diffusion equations. Phys. Rev. E 2002, 66, 046129. [CrossRef] [PubMed]

41. Caputo, M. Linear models of dissipation whose $Q$ is almost frequency independent-II. Geophys. J. Int. 1967, 13, 529-539. [CrossRef]

42. Huang, F.; Liu, F. The space-time fractional diffusion equation with Caputo derivatives. J. Appl. Math. Comput. 2005, 19, 179. [CrossRef]

43. Podlubny, I. Fractional Differential Equations: An Introduction to Fractional Derivatives, Fractional Differential Equations, to Methods of Their Solution and Some of Their Applications; Academic Press: Cambridge, MA, USA, 1998; Volume 198.

44. Zimbardo, G.; Perri, S.; Effenberger, F.; Fichtner, H. Fractional Parker equation for the transport of cosmic rays: Steady-state solutions. Astron. Astrophys. 2017, 607, A7. [CrossRef]

45. Samko, S.; Kilbas, A.; Marichev, O. Fractional Integrals and Derivatives-The Ory and Appli Cations; Gordon and Breach: Longhorne, PA, USA, 1993.

46. Górska, K.; Horzela, A.; Lenzi, E.; Pagnini, G.; Sandev, T. Generalized Cattaneo (telegrapher's) equations in modeling anomalous diffusion phenomena. Phys. Rev. E 2020, 102, 022128. [CrossRef]

47. Halliwell, B.; Gutteridge, J.M. Free Radicals in Biology and Medicine; Oxford University Press: Oxford, UK, 2015.

48. Mainardi, F. Why the Mittag-Leffler function can be considered the Queen function of the Fractional Calculus? Entropy 2020, 22, 1359. [CrossRef]

49. Wright, E.M. On the coefficients of power series having exponential singularities. J. Lond. Math. Soc. 1933, 1, 71-79. [CrossRef]

50. Mainardi, F.; Mura, A.; Pagnini, G. The M-Wright function in time-fractional diffusion processes: A tutorial survey. Int. J. Differ. Equations 2010, 2010. [CrossRef]

51. Gorenflo, R.; Kilbas, A.A.; Mainardi, F.; Rogosin, S.V. Mittag-Leffler Functions, Related Topics and Applications; Springer: New York, NY, USA, 2014. 